Um die berechneten Oerter zu erhalten, wurden für die Sonne Carlinis Tafeln, für den Uranus Bouvard's Tafeln gebraucht, und bei letzterem die Berechnung wieder bis zur tabellar. AR. und Decl. fortgeführt. Dadurch er- gaben sich mit der scheinbaren Schiefe der Ekliptik $=23^{\circ} 27^{\prime} 38^{\prime \prime}, 0$, und mit der Aberr. und Nut. Long. $=31^{\prime \prime}, 2$, und Aberr. Lat, $=0^{\prime \prime}, 0$ folgende Stellungen des Uranus:

\begin{tabular}{|c|c|c|c|c|c|c|c|c|c|c|c|c|c|c|c|c|c|c|}
\hline \multirow{3}{*}{$\underbrace{1826 .}$} & \multicolumn{3}{|c|}{ AR. Uran. appar. } & \multicolumn{3}{|c|}{ Decl, app. } & \multicolumn{3}{|c|}{ Long. geoc. vera } & \multicolumn{3}{|c|}{ Lat. geoc. vera A I } & \multicolumn{3}{|c|}{ Log hel. vera } & \multicolumn{3}{|c|}{ Lat. hel. ver. $\boldsymbol{\Lambda}$} \\
\hline & $\underbrace{\text { Observ. }}$ & tab. & $\underbrace{\text { entr. }}_{\text {eurt. }}$ & & $\underbrace{\text { tab. }}$ & $\underbrace{\text { corrt. }}$ & $\mathrm{abs}$ & $\underbrace{\text { tab. }}$ & corrt. & & $\underbrace{\text { tab. }}$ & $\underbrace{\text { corrt. }}$ & $\underbrace{\text { obs. }}$ & tab. & $\underbrace{\text { corrt. }}$ & & & \\
\hline & $294^{\circ} \prime \prime$ & & & $22^{\circ}$ & & & $292^{\circ}$ & & & & & & $292^{\circ} "$ & & & $0^{\circ}$ & & \\
\hline 1.6 & $20^{\prime} 26,4$ & 33,7 & 7,3 & $5^{\prime} \mathbf{3 0 , 0}$ & 40,2 & 10,2 & $26^{\prime} 37,7$ & 42,6 & 4,9 & $30^{\prime} 36.0$ & 47,3 & 11,3 & 1'19,8 & 24,3 & 4,5 & & 12,7 & 10,7 \\
\hline & 1752,4 & 61,9 & 9,5 & 553,8 & 64,2 & 10,4 & 2413,0 & 19,9 & 6,9 & 3036,1 & 47,9 & 11,8 & 157,5 & 64,0 & 6,5 & 1,9 & 13,1 & 11,2 \\
\hline 8 & 1522,4 & 29,3 & 6,9 & 616,0 & 28,3 & 12,3 & 2152,8 & 56,7 & 3,9 & 3036,0 & 48,5 & 12,5 & 240,8 & 44,5 & 3,7 & 1,6 & 13,5 & 11,9 \\
\hline 9 & 1254,4 & 56,6 & 2,2 : & 646,3 & 52,3 & $6,0:$ & 1932,5 & $|33,2|$ & & 3044,0 & 49,2 & & 323,9 & 24,6 & & 9,1 & 14,0 & \\
\hline 13 & 238,3 & 43,2 & 4,9 & 815,7 & 28,3 & 12,6 & 954,3 & 56,8 & 2,5 & 3037,8 & 51,3 & 13,5 & $\begin{array}{cc}6 & 2,4\end{array}$ & 4,8 & 2,4 & 2,9 & & 12,7 \\
\hline 15 & 5728,7 & 36,6 & 7,9 & $9 \quad 4,4$ & 15,7 & 11,3 & 53,5 & 8,8 & 5,3 & 3039,7 & 52,1 & 12,4 & 720,6 & 25,6 & 5,0 & $\mathbf{4 , 6}$ & 16,4 & 11,8 \\
\hline 18 & 4952,4 & 55 & 3,2 & 1018,8 & 26,4 & 7,6 & 5754,3 & 56,1 & 1,8 & 3045,8 & 53,1 & 7,3 & 923,9 & 25,6 & 1,7 & 2910,6 & 17,6 & 7,0 \\
\hline 19 & 4717,0 & 23,0 & 6,0 & $10,43,4$ & 50,0 & 6,6 & 5528,4 & 32,8 & 4,4 & & 53,5 & 7,5 & $10 \quad 1,9$ & 6,1 & 4,2 & 2910,9 & 18,1 & 7,2 \\
\hline 20 & 4448,0 & 50,0 & 2,0 : & 11 16,8: & $|13,4|$ & $1+3,4:$ & 536,7 & 9,2 & & 3057,8 & 53,9 & & 1043,7 & 46,1 & & & 18,5 & \\
\hline & Cittl & & & & & & & & & & & & & & & & & \\
\hline
\end{tabular}

Zur Bestimmung des Augenblicks der Opposition hat man nun, wenn vorerst an den wahren tabellar. Oertern des

Uranus die so eben gefundene Correction der Tafeln angebracht worden ist, nachstehende Data:

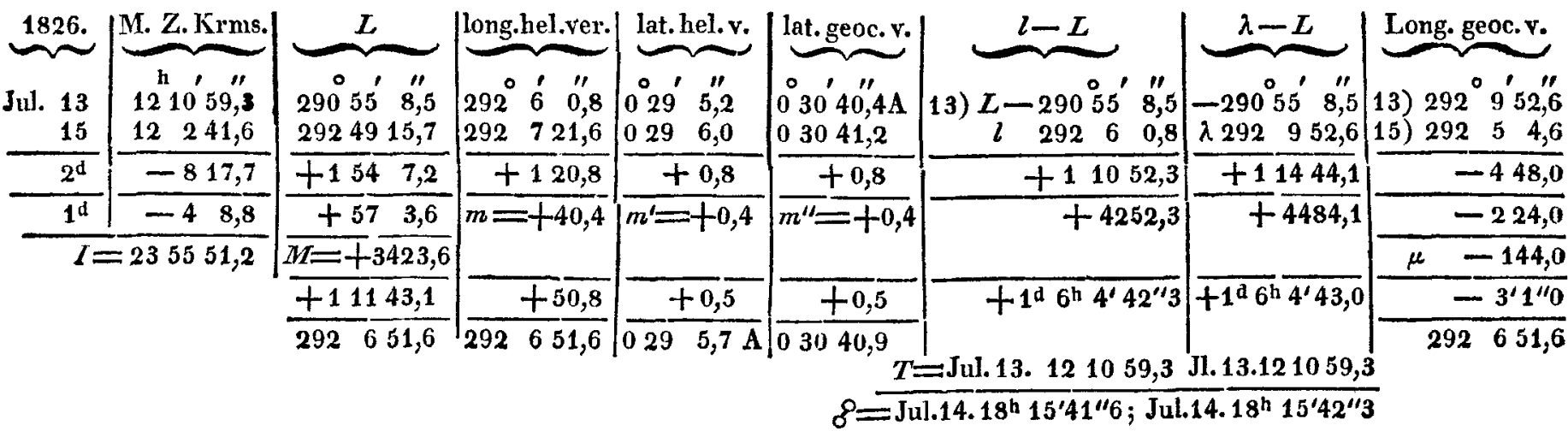

Die Opposition des Uranus erfolgte also im Jalur 1826 den $14^{\text {ten }}$ Juli um $18^{\text {h }} 15^{\prime} 42^{\prime \prime} M$. Z. Krms. als seine beobacht.' wahre helioc. und geoc. Länge $=292^{\circ} 6^{\prime} 51^{\prime \prime}, 6$; heliocentr.
Breite $=0^{\circ} 29^{\prime} 5^{\prime \prime 7}$ A., und geoc. Breite $=0^{\circ} 30^{\prime} 40^{\prime \prime} 9$ A. war. (Der tabell. Ort der Sonne wurde hiebei unverändert beibehalten.)

\title{
Schwarzenbrunner.
}

Auszug aus einem Schreiben des Herrn Professors Nicolai, Directors der Mannh. Sternwarte, an den Herausgeber.

Mannheim 1828. Febr. 8.

..... Die osculirenden Elemente der Juno für die nächst bevorstehende Opposition derselben sind folgende:

Epoche der mittl. Länge 1828. März $250^{\mathrm{h}} \mathrm{M}$. Z. in Mannheim $=160^{\circ} 29^{\prime} 13^{\prime \prime}, 71$.

Tägliche mittlere tropische Bewegung $=813^{\prime \prime}, 69304$

Länge des Perihels $=53^{\circ} 32^{\prime} 13,12$

Excentricitäts-Winkel $=145221,66$
Aufsteigender Knoten $=171^{\circ} 12^{\prime} 11^{\prime \prime}, 18$

Neigung der Bahn $=13318,51$

Log der halben grofsen Axe $=0,4264129$

Epoche, Perihelium und Knoten beziehen sich auf das mittlere Aequinuctium vom $25^{\text {sten }}$ März 1828.

Hieraus ergiebt sich für die nächste Opposition folgende Vorausbestimmung: 
1828 März 25. 7h $13^{\prime} 32^{\prime \prime}$ AI. Z. in Mannhein.

Wahre Länge der Jumo $=185^{\circ} 6^{\prime} 36^{\prime \prime}, 6$

Geocentrische Breite $=+44614,0$

Lichlstärke $=0,02722$.

Bei der letzten Opposition (1826 Oct. 31) war die Lichtstärke $=0,23587$.

Auch beruht auf diesen Elementen nacbstehende Ephemeride für den Lauf der Juno:

\begin{tabular}{|c|c|c|c|c|}
\hline$\underbrace{\mathrm{Macl}}$ & $\begin{array}{l}\text { Febr. } \\
\\
\end{array}$ & 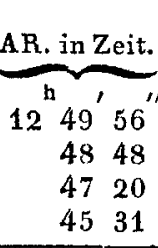 & 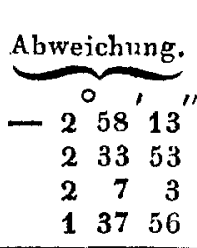 & $\underbrace{\underbrace{}_{0,33473}}_{\begin{array}{c}\text { Log. d. Entf. } \\
\text { v. d. Erde. }\end{array}}$ \\
\hline 8 & $\begin{array}{l}\text { März } \\
\square \\
\square\end{array}$ & $\begin{array}{rrr}12 & 43 & 24 \\
40 & 59 \\
38 & 20 \\
35 & 29 \\
32 & 28 \\
29 & 21 \\
26 & 11 \\
23 & 1\end{array}$ & 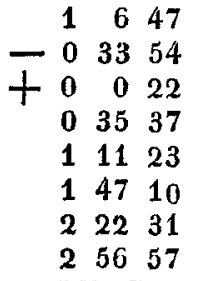 & $\begin{array}{l}0,31236 \\
0,30860 \\
0,30572 \\
0,30379 \\
0,30286 \\
0,30296 \\
0,30410 \\
0,30625\end{array}$ \\
\hline & April & $\begin{array}{rrr}12 & 19 & 55 \\
16 & 56 \\
14 & 7 \\
11 & 29 \\
9 & 5 \\
& 5 \\
6 & 58 \\
5 & 8 \\
& 3 & 37\end{array}$ & $\begin{array}{rrr}3 & 30 & 3 \\
4 & 1 & 25 \\
4 & 30 & 42 \\
4 & 57 & 38 \\
5 & 21 & 59 \\
5 & 43 & 33 \\
6 & 2 & 14 \\
6 & 18 & 2\end{array}$ & $\begin{array}{l}0,30940 \\
0,31352 \\
0,31854 \\
0,32440 \\
0,33102 \\
0,33832 \\
0,34620 \\
0,35458\end{array}$ \\
\hline & May & $\begin{array}{lll}12 & 2 & 25 \\
& 1 & 3.2 \\
& 0 & 59\end{array}$ & $\begin{array}{lll}6 & 30 & 55 \\
6 & 40 & 56 \\
6 & 48 & 11\end{array}$ & $\begin{array}{l}0,36338 \\
0,37251 \\
0,38190\end{array}$ \\
\hline
\end{tabular}

Bei dieser Gelegenheit mache ich mir auch das Verm gnügen, Ihnen dic einzelne Beobachlung mitzutheilen, welche der fast beständig trülse Himmel im vorigen October mir von dem damals von der Sonne zuriickgekehrten letzten Cometen zu erhalten erlaubt hat. Sie ist folgende:

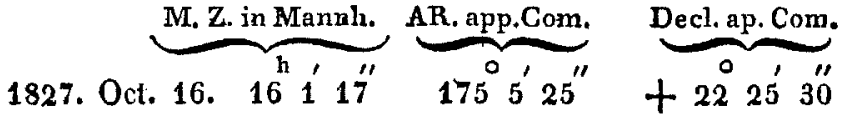

Diese Beolyachtung beruht nur auf zwei, tibrigens recht gut unter einander harmonirenden, Vergleichungen mit 92 Leonis, indem aufsteigender Nebel noch mehrere VerVergleichungen anzustellen verhinderte. Der Comet war bereits sehr lichtschwach, und glich einem kleinen matten Nebelflecke. Es ist mir bis jetzt nicht bekannt geworden, ob derselbe $z u$ jener Zeil auch anderswo beobachtet worden ist. Obige Beobachtung stimmt ̈̈brigens sehr nahe mit der von mir in der Beilage zu Nr. 123. der Astr. Nachr. gegebenen Ephemeride überein, und die ebendaselbst befindliche Parabel bedarf nur sebr unbedentender Correctionen, um eine vollkummene Uebereinstimmung zu erhalten. Somit ist es also noch mehr erwiesen, dars dieser Comet ein ganz anderer ist, als der erste ron 1780.

Bei der Durchsicht meines Cometenbeobachtungs-Buches finde ich auch, dafs ich Ihnen ein paar Beobachtungen des von Pons im August 1826 im Eridanus entdeckten Cometen noch nicht mitgetheilt habe. Es sind diese:

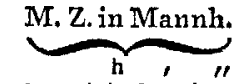

1826. Sept.29. $15{ }^{32} 18^{\prime \prime}$

$$
\begin{array}{lrrrr}
30 . & 16 & 3 & 14 \\
\text { Oct. } & 1 . & 16 & 3 & 46
\end{array}
$$

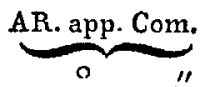

$128 \quad 2314$

$\begin{array}{lll}130 & 6 & 3\end{array}$

1314545

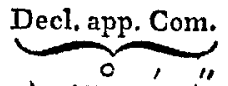

$+17^{\circ} 34^{\prime \prime}$

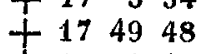

Um die Zeit der letzten Vestaopposition im Decbr. vorigen Jahres habe ich folgende Meridianbeobachtungen dieses Planeten erhalten:

\section{M.Z. in Maunh.}

1827. Dec. 11. 122627,2

- 16. 12115,0

- 17. $1156 \quad 11,9$

- 19. $1146 \quad 5,2$

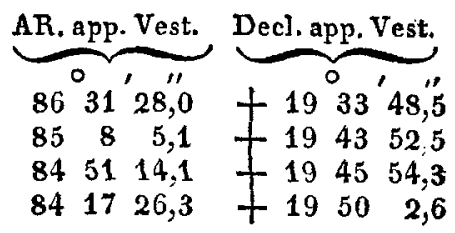

Encke's Ephemeride der Vesta im Astron. Jahrb. für 1829 stimmt auch diefsmal wieder äufserst nahe mit diesen Beobachtungen iiberein. Da derselbe seinen Elementen fortwährend die von mir aus der Juno hergeleitete Jupitersmasse $\frac{1}{1053,924}$ zum Grunde legt, so erprobt sich hiernach letztere Massenbestimmung auch durch die Theorie der Vesta immer mehr und mehr.

B. Nicolai.

\section{Mond $s$ t e r n e f ïr $\mathbf{r} 1828$.}

Herr Dr. Young wird künflig ein Verzeichnifs der mit dem Monde in AR. zu vergleichenden Sterne, berechnen und mit dem Nautical Almanac rertheilen lassen. Er hat die Güte gehabt mir von diesem Verzeïchnisse für 1828 soviel Abdrücke zu versprechen, als nöthig sind, um einem jeden Exemplar der Astron. Nachrichten einen beizulegen: $\mathrm{Da}$ diese Abdrücke aber noch nicht angekommen sind, so lasse ich vurläufig hier die Monate März und April aus einem mir mit der Briefpost übersandten Exemplare abdrucken. 